\title{
Editorial from the Editor in Chief: Inertial Fusion Energy on the Horizon
}

While the current issue was under preparation, the fifth International Conference on Inertial Fusion Sciences and Applications was held at Kobe, Japan, from September 9-14, 2007. This conference has developed into the most important biannual meeting of scientists involved in fusion science and its applications. The topics addressed at this conference are also at the center of interest for readers and authors of Laser and Particle Beams. Inertial Fusion Energy is generally associated with research at major laser facilities like the National Ignition Facility (NIF) in the United States and the project Megajoule in France (Dewald et al., 2006; Kilkenny et al., 2005). Smaller laser facilities like the Vulcan petawatt Laser (Danson et al., 2005), or the Prague Asterix Laser (PALS (Jungwirth, 2005; Batani et al., 2007), and others study specific aspects of laser matter interaction at high intensities. Especially the discovery of high energy electrons and ions accelerated in laser plasma has given a boost to this field (Flippo et al., 2007; Yin et al., 2006; Lifshitz et al., 2006; Glinec et al., 2005 Roth et al., 2005). While laser drivers are expected to achieve ignition and gain in the laboratory, within a few years from now the route to economically feasible inertial fusion energy still remains open. Heavy ion beam drivers from accelerators offer the option of high repetition rate and efficient transformation of electric energy to kinetic energy of the beam ion, which in turn couples its energy in a very efficient way to the bulk matter of the target. Major accelerator laboratories worldwide have been and are currently still engaged in projects to investigate the potential of ion beams as drivers for inertial fusion energy.

It is obvious that significant advances have been made toward inertial fusion. Both large scale laser facilities designed to achieve ignition soon after 2010 are nearing completion. The status of the National Ignition Facility in the U.S and the MegaJoule project in France was the obvious focal point of interest, and is the subject of a large number of recent publications (Giorla et al., 2007; Haynam et al., 2007). However, laser laboratories worldwide are participating in the effort to understand the details of beam matter interaction physics necessary to achieve the conditions of inertial fusion (Laska et al., 2006; Lontano et al., 2006;
Borghesi et al., 2005; Schaumann et al., 2005). The scientific discussion for many years shows (Meyer-ter-Vehn et al., 1990; Funk et al., 1998) and it was confirmed here that the target design is a most crucial issue for the experimental facilities as well as for a future full scale driver (Nobile et al., 2006; Fernandez et al., 2005; Khalenkov et al., 2006; Koresheva et al., 2005).

\section{REFERENCES}

Batani, D., Dezulian, R., Redaelli, R., Benocci, R., Stabile, H., Canova, F., Desai, T., Lucchini, G., Krousky, E., Masek, K., Pfeifer, M., Skala, J., Dudzak, R., Rus, B., Ullschmied, J., Malka, V., Faure, J., Koenig, M., Limpouch, J., Nazarov, W., Pepler, D., Nagai, K., Norimatsu, T. \& Nishimura, H. (2007). Recent experiments on the hydrodynamics of laser-produced plasmas conducted at the PALS laboratory. Laser Part. Beams 25, 127-141.

Borghesi, M., Audebert, P., Bulanov, S.V., Cowan, T., Fuchs, J., Gauthier, JC., MacKinnon, A.J., Patel, P.K., Pretzler, G., Romagnani, L., Schiavi, A., Toncian, T. \& Willi, O. (2005). High-intensity laser-plasma interaction studies employing laserdriven proton probes. Laser Part. Beams 23, 291-295.

Danson, C.N., Brummitt, P.A., Clarke, R.J., Collier, I., Fell, B., Frackiewicz, A.J., Hawkes, S., Hernandez-Gomez, C., Holligan, P., Hutchinson, M.H.R., Kidd, A., Lester, W.J., Musgrave, I.O., Neely, D., Neville, D.R., Norreys, P.A., Pepler, D.A., Reason, C., Shaikh, W., Winstone, T.B., Wyatt, R.W.W. \& Wyborn, B.E. (2005). Vulcan petawatt: Design, operation and interactions at $5 \mathrm{X} 10(20) \mathrm{Wcm}(-2)$. Laser Part. Beams 23, 87-93.

Dewald, E.L., Landen, O.L., Suter, L.J., Schein, J., Holder, J., Campbell, K., Glenzer, S.H., McDonald, J.W., Niemann, C., Mackinnon, A.J., Schneider, M.S., Haynam, C., Hinkel, D. \& Hammel, B.A. (2006). First hohlraum drive studies on the national ignition facility. Phys. Plasmas 13, 056315.

Fernandez, J.C., Hegelich, B.M., Cobble, J.A., Flippo, K.A., Letzring, S.A., Johnson, R.P., Gautier, D.C., Shimada, T., Kyrala, G.A., Wang, Y.Q., Wetteland, C.J. \& Schreiber, J. (2005). Laser-ablation treatment of short-pulse laser targets: Toward an experimental program on energetic-ion interactions with dense plasmas. Laser Part. Beams 23, 267-273.

Flippo, K., Hegelich, B.M., Albright, B.J., Yin, L., Gautier, D.C., Letzring, S., Schollmeier, M., Schreiber, J., Schulze, R. 
\& Fernandez, J.C. (2007). Laser-driven ion accelerators: Spectral control, monoenergetic ions and new acceleration mechanisms. Laser Part. Beams 25, 3-8.

Funk, U.N., Bock, R., Dornik, M., Geissel, M., Stetter, M., Stowe, S., TahiR, N. \& Hoffmann, D.H.H. (1998). High energy density in solid rare gas targets and solid hydrogen. Nuclear Instr. Meth. Phys. Res. 415, 68-74.

Giorla, J., Poggi, F., Galmiche, D., Seytor, P., Quach, R., Cherfils, C., Gauthier, P., Laffite, S. \& Masse, L. (2007). Sensitivity of laser megajoule ignition targets to technological defects. Fusion Sci. Techn. 51, 514-518.

Glinec, Y., Faure, J., Pukhov, A., Kiselev, S., Gordienko, S., Mercier, B. \& Malka, V. 2005. Generation of quasimonoenergetic electron beams using ultrashort and ultraintense laser pulses. Laser Part. Beams 23, 161-166.

Haynam, C.A., Wegner, P.J., Auerbach, J.M., Bowers, M.W., Dixit, S.N., Erbert, G.V., Heestand, G.M., Henesian, M.A., Hermann, M.R., Jancaitis, K.S., Manes, K.R., Marshall, C.D., Mehta, N.C., Menapace, J., Moses, E., Murray, J.R., Nostrand, M.C., Orth, C.D., Patterson, R., Sacks, R.A., Shaw, M.J., Spaeth, M., Sutton, S.B., Williams, W.H., WidmaYer, C.C., White, R.K., YANG, S.T. \& VAN Wonterghem, B.M. (2007). National ignition facility laser performance status. Appl. Opt. 46, 3276-3303.

JungwiRTh, K. (2005). Recent highlights of the PALS research program. Laser Part. Beams 23, 177-182.

Khalenkov, AM., Borisenko, NG., Kondrashov, VN., Merkuliev, YA., Limpouch, J., Pimenov, VG. (2006). Experience of microheterogeneous target fabrication to study energy transport in plasma near critical density. Laser Part. Beams 24, 283-290.

Kilkenny, J.D., Alexander, N.B., Nikroo, A., Steinman, D.A., Nobile, A., Bernat, T, Cook, R., Letts, S., Takagi, M. \& HARding, D. (2005). Laser targets compensate for limitations in inertial confinement fusion drivers. Laser Part. Beams 23, $475-482$.

Koresheva, E.R., Osipov, I.E. \& Aleksandrova, I.V. (2005). Free standing target technologies for inertial fusion energy: Target fabrication, characterization, and delivery. Laser Part. Beams 23, 563-571.

Laska, L., Jungwirth, K., Krasa, J., Krousky, E., Pfeifer, M., Rohlena, K., Ullschmied, J., Badziak, J., Parys, P., Wolowski, J., Gammino, S., Torrisi, L. \& Boody, FP. (2006). Self-focusing in processes of laser generation of highly-charged and high-energy heavy ions. Laser Part. Beams 24, 175-179.

Lifschitz, A.F., Faure, J., Glinec, Y., Malka, V. \& Mora, P. (2006). Proposed scheme for compact Gev laser plasma accelerator. Laser Part. Beams 24, 255.
Lontano, M., Passoni, M., Riconda, C., Tikhonchuk, V.T. \& WEBER, S. (2006). Electromagnetic solitary waves in the saturation regime of stimulated Brillouin backscattering. Laser Part. Beams 24, 125-129.

Mangles, S.P.D., Walton, B.R., Najmudin, Z., Dangor, A.E., Krushelnick, K., Malka, V., Manclossi, M., Lopes, N., Carias, C., Mendes, G., Dorchies, F. (2006). Table-top laserplasma acceleration as an electron radiography source. Laser Part. Beams 24, 185-190.

Meyertervehn, J., Witkowski, S., Bock, R., Hoffmann, D.H.H., Hofmann, I., Muller, R.W., Arnold, R. \& Mulser, P. (1990). Accelerator and target studies for heavy-ion fusion at the Gesellschaft-Fur-Schwerionenforschung. Phys. Fluids B-Plasma Phy. 2, 1313-1317.

Neumayer, P., Bock, R., Borneis, S., Brambrink, E., Brand, H., Caird, J., Campbell, E.M., Gaul, E., Goette, S., Haefner, C., Hahn, T., Heuck, H.M., Hoffmann, D.H.H., Javorkova, D., Kluge, H.J., Kuehl, T., Kunzer, S., Merz, T., Onkels, E., Perry, M.D., Reemts, D., Roth, M., Samek, S., Schaumann, G., Schrader, F., Seelig, W., Tauschwitz, A., Thiel, R., Ursescu, D., Wiewior, P., Wittrock, U. \& Zielbauer, B. (2005). Status of PHELIX laser and first experiments. Laser Part. Beams 23, 385-389.

Nobile, A., Nikroo, A., Cook, R.C., Cooley, J.C., AleXander, D.J., Hackenberg, R.E., Necker, C.T., Dickerson, R.M., Kilkenny, J.L., Bernat, T.P., Chen, K.C., Xu, H., Stephens, R.B., Huang, H., HaAn, S.W., Forsman, A.C., Atherton, L.J., LetTs, S.A., Bono, M.J. \& Wilson, D.C. (2006). Status of the development of ignition capsules in the US effort to achieve thermonuclear ignition on the national ignition facility. Laser Part. Beams 24, 567-578.

Roth, M., Brambrink, E., Audebert, P., Blazevic, A., Clarke, R., Cobble, J., Cowan, T.E., Fernandez, J., Fuchs, J., Geissel, M., Habs, D., Hegelich, M., Karsch, S., Ledingham, K., Neely, D., Ruhl, H., Schlegel, T. \& Schreiber, J. (2005). Laser accelerated ions and electron transport in ultra-intense laser matter interaction. Laser Part. Beams 23, 95-100.

Schaumann, G., Schollmeier, M.S., Rodriguez-Prieto, G., Blazevic, A., Brambrink, E., Geissel, M., Korostiy, S., Pirzadeh, P., Roth, M., Rosmej, F.B., Faenov, A.Y., Pikuz, T.A., Tsigutkin, K., Maron, Y., Tahir, N.A. \& Hoffmann, D.H.H. (2005). High energy heavy ion jets emerging from laser plasma generated by long pulse laser beams from the NHELIX laser system at GSI. Laser Part. Beams 23, 503-512.

Yin, L., Albright, B.J., Hegelich, B.M. \& Fernández, J.C. (2006). $\mathrm{GeV}$ laser ion acceleration from Ultrathin targets: The laser break-out afterburner. Laser Part. Beams 24, 291. 